\title{
Cold atmospheric plasma for COVID-19
}

\author{
Zhitong Chen, Richard E. Wirz \\ Department of Mechanical and Aerospace Engineering \\ University of California, Los Angeles, CA 90095, USA \\ Email: zhitongchen@ucla.edu,wirz@ucla.edu
}

\begin{abstract}
Corresponding Author: Richard E. Wirz, PhD, University of California, Los Angeles, Department of Mechanical \& Aerospace Engineering, 420 Westwood Blvd., Engineering IV, Room 46-147B, Los Angeles, CA, 90095-1596 (wirz@ucla.edu).
\end{abstract}

\begin{abstract}
The recent pandemic has greatly stressed supply chains, treatment modalities, and medical resources. Cold atmospheric plasma (CAP) has been used for a wide range of applications in biomedical engineering due to its many components including electrons, charged particles, reactive oxygen species (ROS), reactive nitrogen species (RNS), free radicals, ultraviolet (UV) photons, molecules, electromagnetic fields, physical forces, and electric fields. In this manuscript, we develop CAP devices for COVID-19. Our manuscript indicates the advantages of highlydeployable CAP devices for both sanitation and treatment, without the need for supply chains of special consumables such as hand sanitizers and the like. We hope that this timely research will help engage the broader community of engineers that wish to help the medical community with this pandemic and to prevent and treat future outbreaks.
\end{abstract}


Coronavirus disease 2019 (COVID-19), an emerging infectious disease caused by severe acute respiratory syndrome coronavirus 2 (SARS-CoV-2), has been inducing a once-in-a-century pandemic, seriously threatening millions, if not billions, of people. ${ }^{1}$ Patients with COVID-19 show manifestations of respiratory tract infection, such as fever, cough, pneumonia, and in severe cases, death. Without a decisive mortality rate yet (currently estimated from $1-6 \%$ ), COVID-19 is believed to be less deadly than SARS $(\sim 10 \%)$ or MERS $(\sim 40 \%)$, while its reproductive number has been estimated to be 2.0 6.57, which is higher than SARS and MERS. As of Mar. 23, 2020, COVID -19 has spread to all continents with multiple epicenters and caused 16,231 deaths out of 372,757 confirmed cases (World Health Organization (WHO), Coronavirus disease 2019 (COVID-19) Situation Report - 64). Although certain physical treatment has been shown to assist patients to fight COVID-19 with their own immune systems, no proven remedies exist so far, inducing high mortality rates especially in senior groups. ${ }^{2}$ Development of effective prevention and treatment is an urgent need, particularly for the life-threatening severe cases and to prevent or curb for future possible outbreaks.

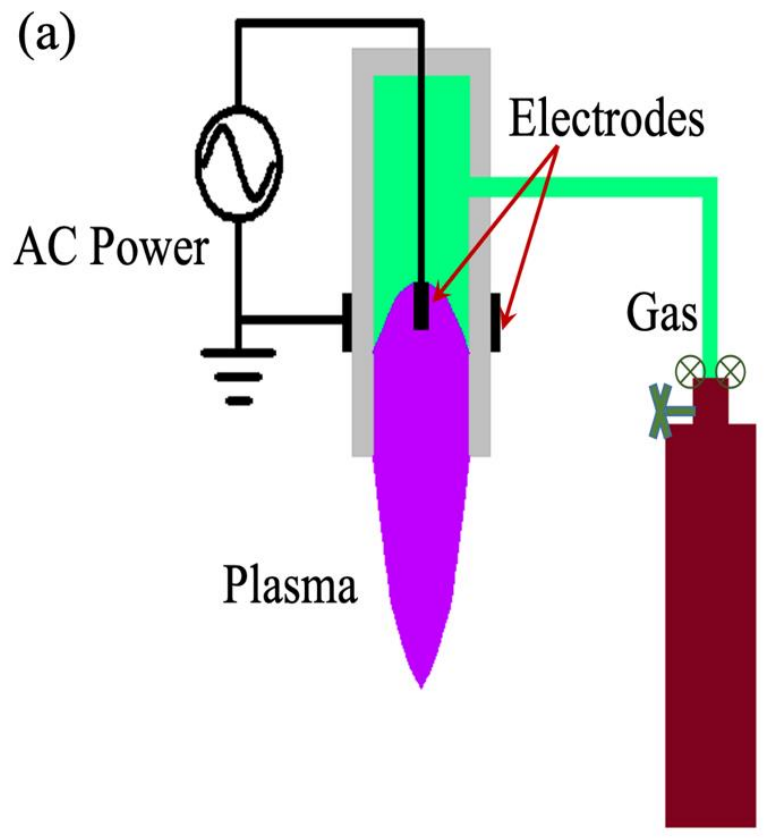

(b)

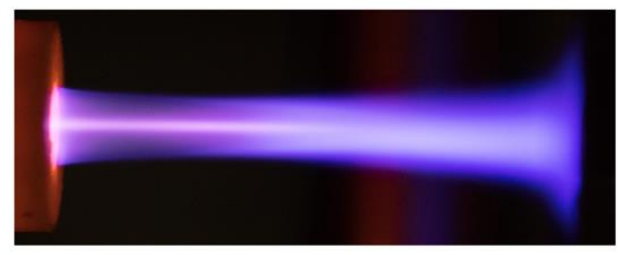

(c)

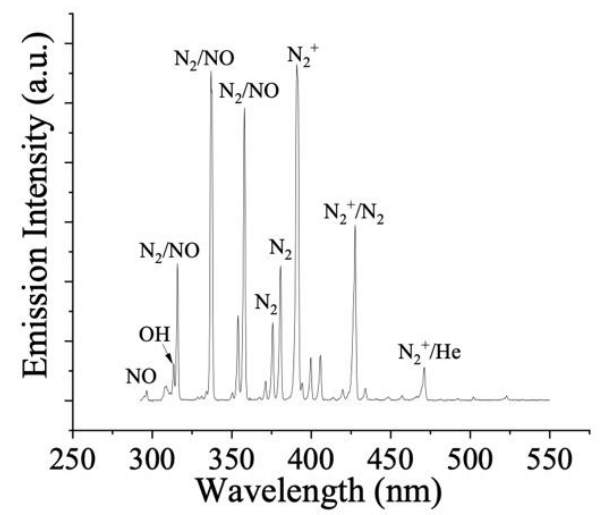

Figure 1. (a) Schematic representation of the cold atmospheric plasma (CAP) setup. (b) Photograph of the He CAP jet. (c) OES spectrum of He CAP jet detected by a fiber-coupled optical spectrometer (LRI-ASEQ Instruments).

Plasma (from Ancient Greek $\pi \lambda \alpha \dot{\sigma} \sigma \alpha$ ) was first described in 1922 by American chemist Irving Langmuir as one of the four fundamental states of matter (i.e., solid, liquid, gas, and plasma). It is the most abundant state of matter, with as much as $99.9 \%$ of the visible universe comprised of plasma. ${ }^{3}$ Recent progress in terrestrially-generated atmospheric plasma has led to the creation of cold atmospheric plasmas (CAP) with ions/heavy particles close to room temperature, thus preventing local heating. Recently, CAP has been used for a wide range of applications in biomedical engineering. ${ }^{4,5}$ The efficacy of CAP is due to its many components that exhibit favorable behavior for biomedical applications, including electrons, charged particles, reactive oxygen species (ROS), reactive nitrogen species (RNS), free radicals, ultraviolet (UV) photons, molecules, electromagnetic fields, physical forces, and electric fields. ${ }^{6}$ Most notably, CAP has been applied to applications such as sterilization, wound healing, coagulation, skin diseases, cancer 
therapy, immunotherapy, and regenerative medicine. ${ }^{7,8}$ CAP device has been developed at UCLA, and its setup schematic representation is shown in Fig. 1(a). Fig. 1 (b) and (c) show helium (He) CAP jet photograph and OES spectrum, respectively. Peaks in Fig. 1 (c) was marked according to reference. ${ }^{9}$

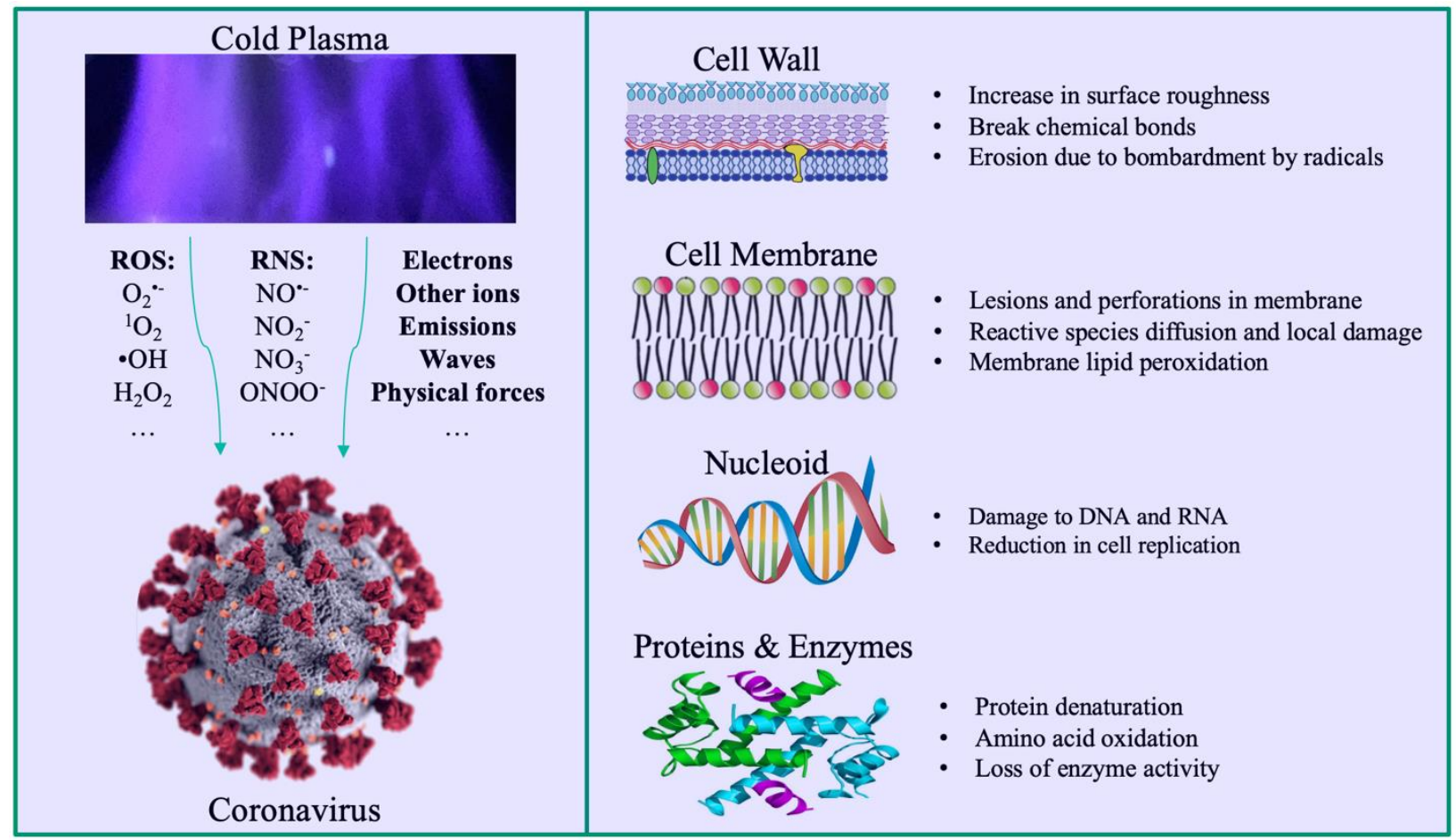

Figure 2. Potential mechanisms for CAP action on COVID-19 leading to loss of functionality and sterilization.

The COVID-19 outbreak has led to dangerous shortages of sterilization capacity at hospitals, businesses, and in homes. Sterilization by CAP is an alternative to conventional and/or traditional sterilization methods. CAP sterilization is caused by plasma-generated reactive species inducing virus leakage and functionality loss. The levels of these reactive species can be adjusted by plasma source design (e.g., electrode orientation and gas feed mechanism), feeding gas types, operating conditions (e.g., electrode voltages and gas flow rates), the nature of the product/substrate, and the micro-organism itself. Studies highlighted the breakage of structurally important bonds, such as $\mathrm{C}-\mathrm{C}, \mathrm{C}-\mathrm{O}$, and $\mathrm{C}-\mathrm{N} .{ }^{10}$ Further, intracellular $\mathrm{pH}$ may be lowered by CAP generated ROS and RNS, as shown in Fig. 1(c), diffusing into the virus, inactivating it due to the absence of $\mathrm{pH}$ homoeostasis. Mass transport through the membrane becomes dysregulated by oxidative damage to membrane lipids. Separately, charged particle accumulating on the virus' surface also damages cell membrane through electrostatic disruption. The electrostatic forces from such an accumulation can exceed the tensile strength of the membrane, leading to its rupture. ROS also play a part in membrane disruption, as they can drive protein denaturation and virus leakage. ${ }^{11}$ Such reactive species can induce oxidation of amino acids, nucleic acids, and unsaturated fatty acid peroxides through interaction with membrane lipids, leading to changes in the membranes' function. Nucleic acids oxidized to 8-hydroxy-2 deoxyguanosine and amino acids oxidized to 2-oxo-histidine. Changes in the membrane directly affect the nucleoid primarily via pore formation and breakdown of the interactions between nucleoid and membrane proteins. ${ }^{12}$ In addition, several other mechanisms for antimicrobial effects also exist, including other reactive atoms and ions, emissions (UV), electrons, 
metastable, and electronically/vibrationally excited molecules. Mechanisms for addressing COVID-19 are driven by the ability of CAP treatment to contribute to virus death including etching virus walls, damaging virus membranes, destruction of genetic materials, and protein/enzyme denaturation, as shown in Fig. 2.
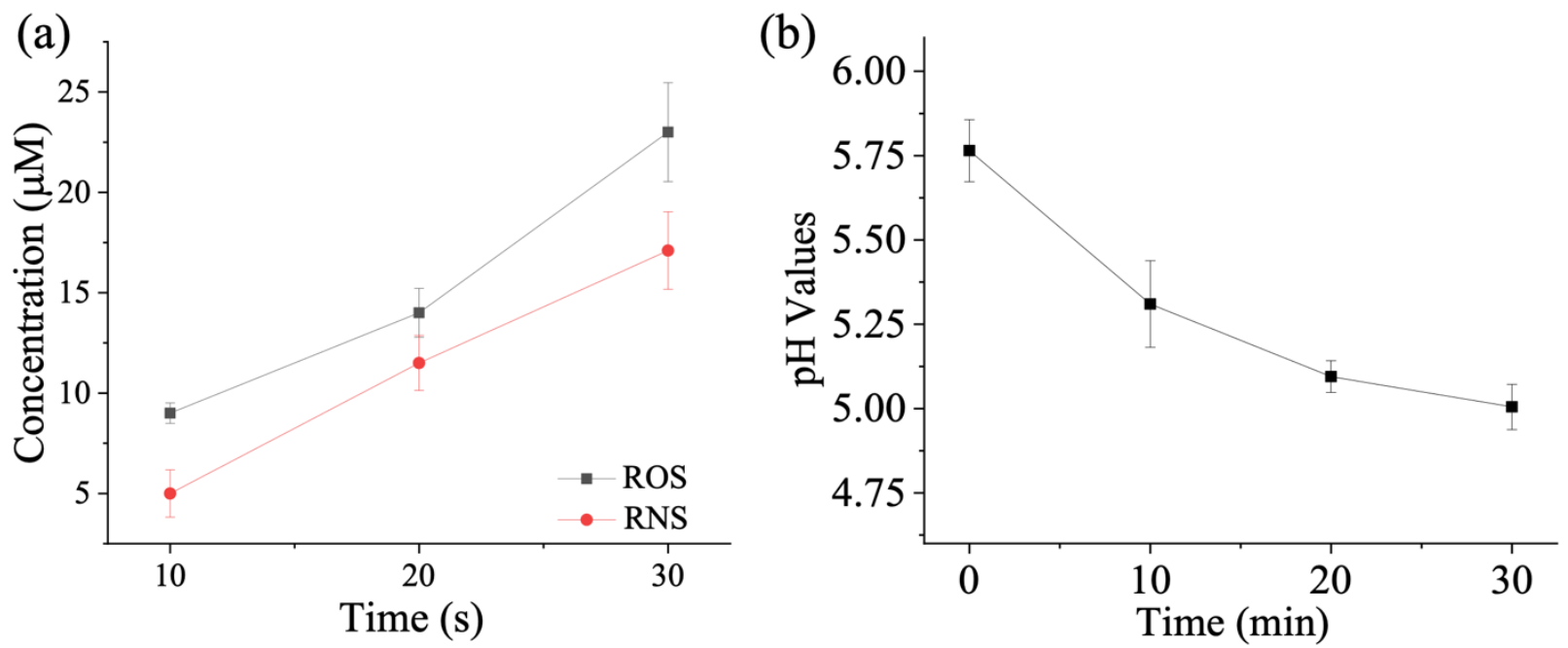

Figure 3. (a) ROS and RNS concentration in He CAP jet treating culture medium measured by a fluorometric hydrogen peroxide assay Kit (Sigma-Aldrich) and a Griess Reagent System (Promega Corporation), respectively. (b) $\mathrm{pH}$ values of DI water treated by He CAP jet changing with treatment time.

The importance of primary infection control measures have been highlighted for COVID-19. Clean hands frequently with alcohol-based hand rub or soap and water has been cited by the WHO as being "one of the most important hygiene measures in preventing the spread of infection". Hands are one of the most frequent transmission routes for many infections because they come in direct contact with known portals of entry for pathogens, such as mouth, nose, and eyes. Recently, CAPactivated medium (liquid) has increasingly be shown as an important approach in the field of plasma biomedicine. ${ }^{13}$ Free radicals and reactive species generated in the atmosphere and at the plasma-medium interface can penetrate side interfaces and enter the so-called "bulk medium" to react or recombine, giving rise to the generation of more stable ROS and RNS (such as $\mathrm{H}_{2} \mathrm{O}_{2}$, $\mathrm{NO}_{2}^{-}$). ROS and RNS concentrations in He CAP jet treatment culture medium show timedependent behavior, as shown in Fig. 3(a). He CAP jet treated DI water resulting into $\mathrm{pH}$ value decreasing, as shown in Fig. 3 (b). According to the Centers for Disease Control (CDC), $\mathrm{H}_{2} \mathrm{O}_{2}$ is a stable and effective disinfectant against viruses. Thus, CAP-activated medium can be used as hand sanitizer to prevent the infection transmission of COVID-19. COVID-19 virus inactivation by CAP-activated mudium results from the combined action of a low $\mathrm{pH}$ and a high positive oxidation reduction potential. CAP-activated mudium can also kill active COVID-19 virus on medical PPE, surfaces, instruments, etc. Such a CAP-based mudium will be of extreme value, processing the functions of sterilizing/disinfection everywhere, such as at home, in the office, public spaces, at entrances of buildings, in hospitals, and in laboratories. This technology can avoid the need for sanitizers that require consumables such as alcohol and are thus susceptible to supply chain disruption.

Another important component of CAP is nitric oxide (NO). NO is a gas that enables diverse biological activities, and can interact with superoxide $\left(\mathrm{O}_{2}^{-}\right)$, forming peroxynitrite $\left(\mathrm{ONOO}^{-}\right)$to 
mediate bactericidal or cytotoxic reactions in turn. ${ }^{14}$ Moreover, NO has played a major role in regulating airway function and in treating inflammatory airway diseases. The beneficial effects of NO inhalation is observed in most patients with severe ARDS (acute respiratory distress syndrome) since it impedes the synthesis of viral protein and RNA. ${ }^{15}$ In addition, organic NO donor, Snitroso-N-acetyl penicillamine, can remarkably obstruct the replication cycle of SARS-CoV. Thus, the NO inhalation can be potential for the treatment of severe COVID-19. Additionally, NO collides with $\mathrm{O}_{2}^{-}$to $\mathrm{ONOO}^{-}$being one of important cytotoxic reactions in plasma cancer therapy. Generally, NO is most prominent for CAP devices that use helium as a supplemental feeding gas. Operators can adjust concentration of NO through changing feeding gas types (nitrogen or mixed gas), operating conditions, and so on. In this way, CAP is one of best sources for controllable NO that can treat severe COVID-19 infected patients.

To better survive in host cells, SARS-CoV-2 employs multiple strategies to avoid immune responses. COVID-19-infected pneumonia patients typically have severe immune abnormalities and risk of cytokine release syndrome (CRS), which results into a decrease of T cells and Natural Killer (NK) cells and an increase of interleukin 6 (IL-6), CD4/CD8 ratio (CD: cluster of differentiation), fever, tissue/organ dysfunction, and an abnormal coagulation function. ${ }^{16}$ Currently, immunotherapy has been proved to an effective treatment to fight COVID-19 infections. The potential mechanism is that inflammatory cells, such as effector T cells and macrophages, accumulate rapidly from peripheral blood by chemokines and release a large number of cytokines into the blood when they kill tumor cells, viruses, or bacteria. Stem cells are hardwired to express antiviral interferon-stimulated genes. In addition, $\beta$-glucan is capable of training both hematopoietic stem cell (HSCs) and myeloid progenitors. These conditioned HSCs and myeloid progenitors can more efficiently ward off inflammatory challenges. ${ }^{17}$ CAP treatment promotes dendritic cell (DC) maturation in the lymph node, where DCs can present the major histocompatibility complexpeptide to T cells. ${ }^{18}$ The subsequent $\mathrm{T}$ cell-mediated immune response can be augmented by the immune checkpoint inhibitors, resulting in enhanced local and systemic antiviral immunity. The adoptive therapy of CAP should be an ideal choice to be employed or combined with other immune modulating agents for COVID-19.

Although the trajectory of COVID-19 outbreak is impossible to predict, timely development and implementation of effective countermeasures are necessary. ${ }^{19} \mathrm{CAP}$ is an alternative to conventional and/or traditional sterilization and treatment methods for COVID-19 due to its causing virus leakage and functionality. Specifically, CAP-activated liquid, containing $\mathrm{H}_{2} \mathrm{O}_{2}$ and exhibiting low $\mathrm{pH}$, is able to kill active COVID-19 virus on hands, medical PPE, surfaces, etc. This prevents the transmission of infection further causing several diseases such as COVID-19, nosocomial food-borne illness, and others. CAP generating NO can treat severe COVID-19 infected patients. Moreover, CAP immunotherapy is an effective treatment to fight COVID-19 infections because it can increase T cells and NK cells to normal levels and recover tissue/organ from dysfunction and coagulation function from abnormal. Until now, there are still no specific antiviral medications approved for COVID-19, however, development efforts are underway. In parallel and during this process, CAP can make its own important contributions to COVID-19 challenges.

In summary, CAP has great potential for preventing the transmission of COVID-19 infections and treating severely infected COVID-19 patients. 
Acknowledgement: This work was supported by grant from Air Force Office of Scientific Research (FA9550-14-10317, UCLA Subaward No. 60796566-114411).

\section{Conflict of Interest Disclosures: None}

\section{References}

1 Burki, T. Outbreak of coronavirus disease 2019. The Lancet Infectious Diseases 20, 292293 (2020).

2 Zhou, F. et al. Clinical course and risk factors for mortality of adult inpatients with COVID-19 in Wuhan, China: a retrospective cohort study. The Lancet (2020).

3 Chen, Z. Development of New Cold Atmospheric Plasma Devices and Approaches for Cancer Treatment, The George Washington University, (2018).

4 Lu, X. et al. Reactive species in non-equilibrium atmospheric-pressure plasmas: Generation, transport, and biological effects. Physics Reports 630, 1-84 (2016).

5 Li, W. et al. Cold atmospheric plasma and iron oxide-based magnetic nanoparticles for synergetic lung cancer therapy. Free Radical Biology and Medicine 130, 71-81 (2019).

6 Chen, Z., Zhang, S., Levchenko, I., Beilis, I. I. \& Keidar, M. In vitro demonstration of cancer inhibiting properties from stratified self-organized plasma-liquid interface. Scientific reports 7, 1-11 (2017).

7 Lin, A. et al. Non-Thermal Plasma as a Unique Delivery System of Short-Lived Reactive Oxygen and Nitrogen Species for Immunogenic Cell Death in Melanoma Cells. Advanced Science 6, 1802062 (2019).

8 Xu, R.-G., Chen, Z., Keidar, M. \& Leng, Y. The impact of radicals in cold atmospheric plasma on the structural modification of gap junction: a reactive molecular dynamics study. International Journal of Smart and Nano Materials 10, 144-155 (2019).

9 Cheng, X. et al. The effect of tuning cold plasma composition on glioblastoma cell viability. PloS one 9 (2014).

10 Yusupov, M. et al. Atomic-scale simulations of reactive oxygen plasma species interacting with bacterial cell walls. New Journal of Physics 14, 093043 (2012).

11 Moisan, M. et al. Low-temperature sterilization using gas plasmas: a review of the experiments and an analysis of the inactivation mechanisms. International journal of Pharmaceutics 226, 1-21 (2001).

12 Scholtz, V., Pazlarova, J., Souskova, H., Khun, J. \& Julak, J. Nonthermal plasma-A tool for decontamination and disinfection. Biotechnology advances 33, 1108-1119 (2015).

13 Chen, Z. et al. A novel micro cold atmospheric plasma device for glioblastoma both in vitro and in vivo. Cancers 9, 61 (2017).

14 Zhang, L. \& Liu, Y. Potential interventions for novel coronavirus in China: A systematic review. Journal of medical virology (2020).

15 Lei, C. et al. Protocol of a randomized controlled trial testing inhaled Nitric Oxide in mechanically ventilated patients with severe acute respiratory syndrome in COVID-19 (SARS-CoV-2). medRxiv (2020).

16 Wang, W., He, J. \& Wu, S. The definition and risks of Cytokine Release Syndrome-Like in 11 COVID-19-Infected Pneumonia critically ill patients: Disease Characteristics and Retrospective Analysis. Medrxiv (2020). 
17 Wu, X. et al. Intrinsic immunity shapes viral resistance of stem cells. Cell 172, 423-438. e425 (2018).

18 Chen, G. et al. Transdermal cold atmospheric plasma-mediated immune checkpoint blockade therapy. Proceedings of the National Academy of Sciences 117, 3687-3692 (2020).

19 Paules, C. I., Marston, H. D. \& Fauci, A. S. Coronavirus infections-more than just the common cold. Jama 323, 707-708 (2020). 